\title{
A Model of Disturbed Eating Behavior in Men: The Role of Body Dissatisfaction, Emotion Dysregulation and Cognitive Distortions
}

Andrea Wyssen ${ }^{a},{ }^{*}$ Jana Bryjova ${ }^{a},{ }^{*}$ Andrea Hans Meyer ${ }^{b}$ \& Simone Munsch ${ }^{a * *}$

a. Institute of Psychology, Department of Clinical Psychology and Psychotherapy, University of Fribourg, Rue de Faucigny 2, 1700 Fribourg, Switzerland, andrea.wyssen@unifr.ch, jana.bryjova@unifr.ch, simone.munsch@unifr.ch

b. Institute of Psychology, Department of Clinical Psychology and Epidemiology, University of Basel, Missionsstrasse 62A 4055 Basel, Switzerland, andrea.meyer@unibas.ch

*shared first authorship

**Correspondence to:

Simone Munsch, PhD

Universität Fribourg

Department für Psychologie

Klinische Psychologie und Psychotherapie

2, Rue de Faucigny

CH-1700 Fribourg/Switzerland

TEL: +41 2630076 57/55

FAX: +41263009712

simone.munsch@unifr.ch

(C) 2016. This manuscript version is made available under the Elsevier user license http://www.elsevier.com/open-access/userlicense/1.0/ 


\section{A Model of Disturbed Eating Behavior in Men: The Role of Body Dissatisfaction, Emotion Dysregulation and Cognitive Distortions}

\section{Introduction}

Lifetime prevalence of eating disorders (EDs) in men of the general population range from 0.2 to $0.4 \%$ for anorexia nervosa, 0.1 to $0.9 \%$ for bulimia nervosa and 1.0 to $1.1 \%$ for binge-eating disorder (Allen et al., 2013; Hudson et al., 2007; Preti et al., 2009; Raevuori et al., 2014). In contrast to the relatively low prevalences of full-blown EDs, symptoms of disordered eating on a subclinical level are more frequent in men (Woodside et al., 2001). Recurrent binge-eating or extreme dietary restraints were reported by up $7.7 \%$ of the general male population. Further dysfunctional eating and weight/shape control behaviors include excessive exercising and steroid use, which are especially prevalent among men with body image disturbances. Up to $13.5 \%$ of men further reveal marked overvaluation of shape and/or weight (for an overview see Mitchison and Mond, 2015), which is a core common feature of current comprehensive trans-diagnostic cognitive behavioral models of ED in men (Cooper and Fairburn, 2011; Dakanalis et al., 2014a).

Body dissatisfaction (BD) is frequently used in this context as a term to describe an individuals' body-related negative self-evaluation. This term does not necessarily refer to clinical symptoms of a disturbed body image or eating behavior, but refers to the degree individuals are concerned about or dissatisfied with their appearance. Representative studies show that BD is widely spread among both genders. Around $60-80 \%$ of men report being dissatisfied with their overall body or parts of their body (Fiske et al., 2014; Thompson and Cafri, 2007). Men's concerns regarding their body image seem two-dimensional, referring to either an increase in muscularity and/or weight loss/body fat reduction. Accordingly, BD in men may either result in muscularity increasing behavior or in efforts to lose weight (e.g., Tylka, 2011). Importantly, etiological models consistently found BD to be a causal risk factor for ED symptomatology in women and men (Dakanalis et al., 2014a; Raevuori et al., 2014; Stice, 2002; Stice and Shaw, 2002). In a longitudinal study, Neumark-Sztainer and colleagues (2006) found that BD among US-male adolescents predicted dysfunctional weight control strategies such as 
fasting or inappropriate use of food supplements and binge eating. Other studies confirmed these results and showed that $\mathrm{BD}$ is associated with other health risk behaviors aiming at increasing muscularity and reducing body fat, such as steroid abuse and supplement intake (Blashill, 2014; Brower et al., 1994; Cafri et al., 2005; Ricciardelli and McCabe, 2003). Most recently, Dakanalis and colleagues (2016) found that $\mathrm{BD}$, negative affect and low self-esteem predicted binge-eating and compensatory behaviors in a longitudinal study with over 2500 male college students.

In sum, the association between BD and ED pathology in men is well established. However, mechanisms relating the common phenomenon of $\mathrm{BD}$ to the development of an ED still remain unclear. The most established model, addressing potential mediators, is Stice's (2001) dual pathway model of bulimic pathology, which was proposed and evaluated in cross- and longitudinal studies with females samples (Stice, 2001; Stice et al., 2011). The model suggests that BD contributes to dieting behavior (path 1) and negative affect (path 2), which in turn increases the risk of developing ED pathology in terms of restricting, dieting, binge eating and compensatory behaviors as an attempt to control weight and shape and to regulate negative emotions (Stice, 2001). In recent years, a considerable number of studies were conducted to further clarify the relationship between BD and ED pathology as well as the mediating and moderating psychological mechanisms involved on male samples (e.g., Dakanalis et al., 2015a; Dakanalis et al., 2014b; Dakanalis et al., 2015c). Lavender and Anderson (2010), for example, found in their sample of young male college students, that those who experience pronounced negative mood due to their BD were more likely to engage in maladaptive behaviors, such as binge eating, purging and excessive exercise, especially when they reported to not being able to accept or regulate their negative mood states. In accordance with this results, Dakanalis and colleagues (2015a) found emotional dysregulation to amplify the relationship between BD and ED symptomatology in a sample of 557 men at the age of $18-28$ years.

Based on a cognitive-behavioral approach relating BD to ED pathology, there is a need of further data on the role of cognitive distortion to complete the current models, e.g. the dual pathway model (Culpert et al., 2015). Distorted cognitions are characterized by skewed and non-veridical 
thoughts related to affective experiences and behaviors that have been linked to psychological problems and mental disorders (Rachman and Shafran, 1999). Under certain conditions, individuals may have difficulties to separate themselves from their negative body-related thoughts which results in a so-called "thought fusion", a concept that has particular importance in the Acceptance and Commitment Therapy (ACT) (Hayes et al., 1999). Thought fusion results in self-identification with one's own thoughts, and has been associated with cognitive inflexibility, which seems to be an important etiological factor in EDs (Merwin et al., 2011).

Derived from the original concept of "Thought-Action Fusion" (TAF) in obsessive-compulsive disorder (OCD) (Shafran et al., 1996), Shafran and colleagues (1999) proposed "Thought-Shape Fusion" (TSF) as a specific cognitive distortion mechanism in ED. TSF describes specific distorted cognitions with respect to one's own body triggered by the imagination of eating fattening/forbidden food. Not only that TSF-like distorted cognitions evoke negative feelings about one's own body (e.g., Coelho et al., 2013), they are also directly related to dysfunctional behaviors, such as the urge to engage in body checking or to restrict food intake (Shafran et al., 1999). So far, associations between TSF and the severity of ED symptoms as well as general psychopathology have been demonstrated predominantly in studies with female samples (Shafran and Robinson, 2004; Shafran et al., 1999). On the other hand, we have found only one study investigating TSF with male participants from an Australian community sample demonstrating a significant association between TSF and ED pathology as well as BD (Dubois et al., 2013).

The TSF concept has been described for three theoretically postulated factors: Likelihood (the mere thought of eating fattening/forbidden food leads to the sensation that a subsequent weight gain is more likely), Moral (the thought of eating fattening/forbidden food is morally as wrong as the included act) and Feeling (the thought of eating fattening/forbidden food evokes feelings of fatness) (Shafran et al., 1999). Although people, who are highly susceptible to TSF, probably know on a cognitive level that merely thinking about eating certain foods cannot influence their body shape or weight, they nevertheless feel on an emotional level that this might be the case (Shafran et al., 
1999). However, the theoretically postulated three-dimensional factor structure of the TSF questionnaire has not been found in empirical validation studies yet.

TSF has also been investigated in experimental studies: It has been shown that the imagination of eating fattening/forbidden foods may directly lead to distorted cognitions which influenced selfperception, in terms of feeling fat, concerns about weight gain and the experience of moral wrongdoing. This effect has been demonstrated mostly in female samples (Coelho et al., 2008; Coelho et al., 2010; Radomsky et al., 2002).

To conclude, the current research including prospective studies suggests a strong association between BD and ED pathology in men (e.g., Dakanalis et al., 2015a; Dakanalis et al., 2015c; Lavender and Anderson, 2010). The present study reevaluates the role of negative emotionality according to the dual pathway model of Stice (2001) by testing the mediating effect of emotion dysregulation. While in Stice's model, behavioral (i.e. dietary restraint) and emotional (i.e. negative affect) components were considered as mediators of the association between $\mathrm{BD}$ and $\mathrm{ED}$, we chose to test a model that includes in addition to an emotional component, a novel cognitive construct, the Thought-Shape Fusion (TSF), that has theoretical and empirical support in relation to disturbed eating (e.g., Fairburn et al., 2003; Shafran and Robinson, 2004). Furthermore, instead of negative affect, we included a related construct of emotion dysregulation, which should not only reflect deficits in the modulation of emotional arousal, but also in the awareness, understanding, and acceptance of emotions, as well as the ability to act in desired ways regardless of emotional state (Gratz and Roemer, 2004).

On the basis of the literature reviewed, we expected BD to predict emotion dysregulation, which in turn would predict disturbed eating and compensatory behavior in men. Similarly, we expected TSF to mediate the association between $B D$ and DECB in men. Thus, the direct effect between the $B D$ and $D E C B$ should become smaller, when including TSF and emotion regulation as mediators into the model (see figure 1). 
We tested our model in a cross-sectional online study using a sample of 123 men aged between 18-37 years and living in Switzerland. Since previous studies investigated BD and eating pathology in slightly more homogenous and younger male populations (e.g., Dakanalis et al., 2015a; Neumark-Sztainer et al., 2006), we decided to controll for the effect of participant's age in the statistical analyses. Furthermore, we controlled for depressive symptoms to be able to rule out the influence of this marker of general psychopathology (Gotlib, 1984). Thirdly, we controlled for BMI in our analyses, since there is a known influence of BMI on body fat and muscularity dissatisfaction, as well as on disordered eating in undergraduate men (Tylka, 2011).

\section{Method}

\subsection{Participants and procedure}

Participants were recruited for the survey through mailing lists, flyers and an advertisement on the website of the University of Fribourg, Switzerland. The online survey was carried out at the Department of Clinical Psychology at the University of Fribourg and took approx. 40 to 50 minutes to complete. The local ethical committee approved the study design, procedure, and materials. Students received course credits as compensation. Other non-student participants were not compensated for their participation; however they were offered the possibility to receive a summary of the results. Altogether 123 men were registered for the online survey. The mean age was 23.7 $(S D=3.2)$ years and the mean BMI of participants was $23.3(S D=3.1)$. One third of the sample had Swiss nationality, $63.4 \%$ was German and $3.3 \%$ were of other nationality. More than two thirds (68.3\%) of the participants were students, $27.6 \%$ were employed, and $4.1 \%$ did not indicate their occupation.

\subsection{Measures}


Standardized questionnaires in German were presented via an online survey platform (Umfrageonline; Enuvo) during 2012-2014. The original format and content of the questionnaires were not altered.

Body Mass Index (BMI): BMI ( $\left.\mathrm{kg} / \mathrm{m}^{2}\right)$ was calculated relying on self-reported body weight and height as assessed in the EDE-Q. A BMI below 18.5 refers to underweight, above 25 indicates overweight, and above 30 obesity.

Depressive symptoms (BDI-II): To assess correlates of general psychopathology we measured depressive symptoms using the Beck Depression Inventory (BDI-II), which consists of 21 items and measures the severity of depressive symptoms (e.g., lack of joy, feelings of guilt and sadness) during the previous two weeks (Beck et al., 1996). The instrument has good validity and reliability (Beck et al., 1996; Hautzinger et al., 2009). Cronbach's alpha of the German version is $\geq .84$ (Kühner et al., 2007), the corresponding value in this sample was .90 .

Body dissatisfaction (BD): To assess body shape preoccupations the short-version of the Body Shape Questionnaire (BSQ-8C; original English version by Evans and Dolan, 1993; German version by Pook et al., 2002) was used. This self-report questionnaire relates to the last four weeks and includes items such as "Has seeing your reflection (e.g., in a mirror or shop window) made you feel bad about your shape?" The approved shortened form of the BSQ shows good convergent and discriminant validity as well as reliability (Evans and Dolan, 1993). While most studies investigated the validity of the BSQ scales on female samples (e.g., Pook et al., 2008), fewer studies addressed the validity of BSQ in mixed-gender populations with satisfactory results (e.g., Di Pietro and Silveira, 2009; Probst et al., 2009; Rosen et al., 1996). Cronbach's alpha for this sample was .90. Values below 19 indicate no concerns with shape, scores from 19 to 25 refer to mild concerns with shape, and for values above 25 moderate to marked concerns with shape have to be considered (Evans and Dolan, 1993).

Disturbed Eating and Compensatory Behavior (DECB): In order to avoid a conceptual overlap between the variable $\mathrm{BD}$ and the weight and shape concerns from the Eating Disorder Examination Questionnaire (EDE-Q; original English version by Fairburn and Beglin, 1994; German version by 
Hilbert and Tuschen-Caffier, 2006), we created a new variable named Disturbed Eating and Compensatory Behavior (DECB), in which items conceptually related to the $\mathrm{BD}$ are omitted. The DECB scale involves following items from the EDE-Q questionnaire: items 1-5 that describe symptom of ED pathology such as restrained eating (e.g., "Have you been deliberately trying to limit the amount of food you eat to influence your shape or weight?"), the frequency of binge-eating over the past 28 days (item 15), frequency of self-induced vomiting over the past 28 days (item 16), frequency of taking laxatives over the past 28 days (item 17) and frequency of excessive exercising over the past 28 days (item 18). Thus, the items assessing the shape and weight concerns from the EDE-Q scale were omitted in the DECB scale. Items 1-5 were scored on a scale from 0 to 6 ; items $15-18$ were coded based on the given occurrences of binge-eating (number of days over the past 28 days), as well as purging, exercising and use of laxatives (number of events over the past 28 days). The coding has been done as follows: 0 days $=0,1-5$ days $=1,6-12$ days $=2,13-15$ days $=3,16-22$ days $=4,23-$ 27 days $=5,28$ days and more $=6$ ). The EDE-Q has demonstrated good psychometric properties and has shown to be suitable to detect significant symptoms of EDs in individuals of the general population (Mond et al., 2004). Cronbach's alpha for the global score is .97 and subscale values range from .85 to .93 (Hilbert et al., 2007). Corresponding values in our sample were .82 for the global score and .80 for the new DECB scale.

Emotion dysregulation: The Difficulties with Emotion Regulation Scale (DERS; Gratz and Roemer, 2004) (German version by Ehring et al., 2008) is a 36-item self-report questionnaire assessing six dimensions of emotion regulation: none acceptance of emotions (e.g., "When I'm upset, I feel guilty for feeling that way."), difficulties in engaging in goal directed behavior (e.g., "When I'm upset, I have difficulty concentrating."), impulse control difficulties (e.g., "When I'm upset, I lose control over my behaviors."), lack of emotional awareness (e.g., "I am attentive to my feelings."), limited access to emotion regulation strategies (e.g., "When I'm upset, I believe that I'll end up feeling very depressed."), and lack of emotional clarity (e.g. "I have difficulty making sense out of my 
feelings."). The total DERS showed good validity and internal consistencies $(\alpha=.93$ ) (Gratz and Roemer, 2004). Cronbach's alpha of the DERS global score in the present sample was .93.

Cognitive distortions (TSF): The Short Trait Thought Shape Fusion Scale (TSF trait scale; Coelho et al., 2013) provides an assessment of eating and body image related cognitions. Coelho and colleagues (2013) developed a short version of the original trait TSF scale (Shafran et al., 1999) consisting of 14 items assessing the TSF concept (e.g., "I feel fatter after thinking about eating fattening/"forbidden" foods.") and 4 items to measure the clinical impact (e.g., "Generally, to what extent do thoughts about "forbidden" foods affect you, or interfere with your daily life?"). The scale was translated into German and back translated (German version, available from the authors). Cronbach's alpha for the 14-items scale "concept" in this sample was .87. This scale has not yet been validated for the male population.

\subsection{Data analysis}

We conducted a multiple mediation model with two mediators operating in parallel and being allowed to correlate with each other (see model 4 in Hayes, 2012). In this model, the variable body dissatisfaction (BD) was the independent variable, dysfunctional eating and compensatory behavior (DECB) the dependent variable, and variables thought-shape fusion and emotion dysregulation the two mediators (see Figure 1). Participants' age, BMI as well as depressive symptoms were included as covariates since they were previously shown to be associated with disturbed eating. Variables BD, TSF, DECB and the covariates were all log-transformed, and the variable emotion dysregulation was square-root transformed. Confidence limits were estimated using bootstrapped samples $(N=5000)$ with adjusted bootstrap percentiles (BCa). Statistical analyses were conducted with the statistical software R version 3.1.3 (2015), including the R package “lavaan" (Rosseel, 2012).

\section{Results}

\subsection{Preliminary results}


Descriptives of the assessed variables are listed in Table 1. Mean global score of the EDE-Q was 0.53 $(S D=0.63)$. Three participants $(2.4 \%)$ scored higher than 2.09 on the EDE-Q global score, which refers to elevated ED psychopathology when compared to a healthy control sample of men (Lavender et al., 2010). According to the cut-off values for the BSQ, 14 participants (11.4\%) reported elevated BD. Twelve participants (9.8\%) scored above the cut-off of the BDI-II. Regarding the dependent variable DCEB, 18 (14.6\%) participants scored above the mean value plus one standard deviation $(M=0.35$, $S D=0.61)$, which refers to a comparatively distinct tendency to disturbed eating and compensatory behavior in this subgroup. Eighteen participants (12.8\%) did not finish the online survey and were not included into the analyses. Men who terminated the survey prematurely scored significantly higher on the shape concern scale of the Eating Disorder Examination Questionnaire (EDE-Q) $(t(140)=-2.75$, $p=0.013)$, as well as in global symptoms of ED pathology according to the EDE-Q $(t(140)=-2.30$, $p=0.033$ ) compared to the completers. No significant differences were found regarding BMI, age and depressive symptoms between completers and non-completers.

Insert Table 1 here

\subsection{Main results}

In the following, we report unstandardized coefficients including standard errors and $p$-values in the text, whereas standardized coefficients and additional statistics are reported in table 2 . The total effect of BD on DECB was significant ( $c=0.37, S E=0.07, p<0.001)$. Moreover, BD was significantly associated with increased body-related cognitive distortions (TSF) ( $\left.a_{1}=1.49, S E=0.25, p<0.001\right)$, as well as with increased emotion dysregulation $\left(a_{2}=1.42, S E=0.35, p<0.001\right)$. Furthermore, increased level of TSF was significantly associated with increased DECB $\left(b_{1}=0.09, S E=0.03, p<0.001\right)$, but there was no significant association between emotion dysregulation and DECB $\left(b_{2}=-0.03, S E=0.02\right.$, 
$p=0.09)$. The direct effect of BD on DECB, controlling for TSF and emotion dysregulation, was smaller compared to the total effect, but remained significant $\left(c^{\prime}=0.29, S E=0.08, p=0.001\right)$. To quantify the difference between the total and the direct effect, indirect effects of the two mediators on DECB were assessed. While the indirect pathway of BD on DECB through TSF was significant $\left(a_{1}{ }^{*} b_{1}=0.13\right.$, $S E=0.04, p=0.001$, the indirect pathway of BD on DECB through emotion dysregulation was not $\left(a_{2}{ }^{*} b_{2}=-0.05, S E=0.03, p=0.12\right)$. Thus, the pathway via TSF was of higher magnitude than the pathway via emotion dysregulation $\left(a_{1}{ }^{*} b_{1}-a_{2}{ }^{*} b_{2}=0.18, S E=0.05, p=0.001\right)$. The correlation between the two mediators TSF and emotion dysregulation was $0.10(p=0.076)$. The mediator model explained $43 \%$ of the total variance in DECB.

Insert Table 2 here

Insert Figure 1 here

\section{Discussion}

In order to better understand the mechanisms underlying the relationship between $\mathrm{BD}$ and DECB, we proposed a model of disturbed eating behavior in men, in which emotion dysregulation and the susceptibility to body-related cognitive distortions such as TSF act as mediators. We tested the model in a cross-sectional online survey on a sample of young men living in Switzerland, and included participant's age, BMI and depressive symptoms as covariates.

Whereas none of the covariates such as $\mathrm{BMI}$, age and depressive symptoms were related to eating pathology, we found a significant positive association between $B D$ and $D E C B$, which is in accordance with the proposed theoretical model. Our results thus underline previous findings linking 
BD to disturbed eating and unhealthy weight and shape regulation strategies in men (e.g., Dakanalis et al., 2016; Neumark-Sztainer et al., 2006). In our sample of young male students, only $1.6 \%$ of participants were obese (BMI > 30), 9.8\% showed clinically relevant depressive symptoms (BDI > 13; Hautzinger et al., 2009) and 13\% exceeded a critical value regarding emotion regulation difficulties (> 99; Gratz and Roemer, 2004). In order to obtain more generally interpretable results, it would be desirable to replicate our model in a less homogenous sample with a greater proportion of men with clinically relevant psychopathology.

Consistent with our hypothesis, there was a significant mediating effect of TSF on the association between $\mathrm{BD}$ and $\mathrm{DECB}$ in a sample of young men. Thus increased BD predicted an enhancement of cognitive distortion, which in turn predicted higher levels of disturbed eating. This emphasizes the importance of TSF in relation to disordered eating also in men. The susceptibility to body-related distorted cognitions may be a correlate of a certain cognitive inflexibility in the course of cognitive fusion. That is, high BD does not necessarily lead to DECB if an individual is capable to dissociate oneself from the intrusive thoughts which are triggered by certain stimuli such as food items and body ideals. In contrast, the tendency to stick to such thoughts may favor pathological eating and body-related behavior. Since exposure to fattening foods and body ideals occur repeatedly on a daily basis, future studies should emphasize the role of cognitive processes, such as TSF, and especially focus on resulting behavioral consequences such as the urge to diet or frequent body checking (e.g., Coelho et al., 2008; Wyssen et al., 2016). Finally, a more profound knowledge about the effects of TSF and related cognitive inflexibility on ED pathology have the potential to further improve therapeutic interventions (such as ACT; Hayes et al., 1999).

Previous literature suggested the role of disinhibited eating as an attempt regulate negative affective states (Heatherton and Baumeister, 1991), which was supported by data from female (Svaldi et al., 2012) and male (Lavender and Anderson, 2010) samples. In contrast to our expectations, which were based on these results, our data could not confirm the mediating effect of emotional dysregulation on the association between $\mathrm{BD}$ and $\mathrm{DECB}$. While this is surprising, the non- 
significant result does not necessarily rule out the existence of such, as recently pointed out by Dienes (2011). More likely, several factors might have contributed to the unexpected result, such as the fact a different age range as well as ethnic composition of our sample compared to that in the study by Lavender and Anderson (2010). Additionally, only $2.4 \%$ of men in our study reported elevated ED psychopathology, and $11.4 \%$ reported clinically relevant BD according to the cut-off values reported elsewhere (e.g., Lavender et al., 2010). Since the examined sample included individuals indicating no ED behaviors as well as no $\mathrm{BD}$, the range over which these variables were measured may have been somewhat restricted, thereby decreasing the power to detect associations. It further remains unclear, whether men's tendency to engage in automatic and non-conscious emotion regulation processes (Nolen-Hoeksema, 2012), which are not addressed by the DERS, might represent a more gender-specific emotion regulation attribute relating to ED symptoms. Additionally, empathizing ruminative thinking about one's own body shape, as a correlate of dysfunctional emotion regulation tendencies, may lead to further clarification. This is supported by the recent finding that rumination induction increased ED pathological behavior in women suffering from EDs (Naumann et al., 2015).

The present study has several limitations that warrant attention. First of all, the cross-sectional design of our study does not allow drawing any conclusion about the causality of the relationship among the variables involved and the need for longitudinal studies is stressed. As our mediation model was just identified, we were not able to test whether it fitted the data better than competing models, in which the direction of the paths were reversed. This is because any variant of a justidentified model (i.e. one with reversed directions of the paths) would also be just identified and hence equivalent to our model. In this respect our results remain somewhat tentative and need further validation using different male samples. Secondly, the sexual orientation of men in our sample was not assessed. Evidence has shown that homosexual compared to heterosexual male adolescents and young adults tend to be more dissatisfied with their appearance (e.g., Jankowski et al., 2014). It would therefore be interesting to investigate whether the coefficients in our model 
differ depending on men's sexual orientation. Sociocultural factors, such as exposure to mass media presenting unrealistic male body ideals and the associated degree of internalization of the ideal as well as the perceived pressure to conform to this ideals may have further impact on the variables in our model and should be included in future models (Barlett et al., 2008). Another drawback of this study is the use of some questionnaires that have been originally developed for women and then adapted for men (such as the BSQ), as they focus on the drive for thinness and possibly neglect specific male concerns about losing body fat and gaining muscles (e.g., Dakanalis et al., 2015b; McCreary et al., 2004; Tylka et al., 2005).

Compared to other online-studies in which dropout rates amount up to $42.7 \%$ (e.g., Jankowski et al., 2014), the proportion of male participants who did not complete all measures (12.8\%) in our study is relatively low. However, those who terminated the survey prematurely, scored significantly higher on the shape-concern scale of the EDE-Q, as well as in global symptoms of ED pathology according to the EDE-Q compared to the completers. This may refer to an aversive experience in men with higher ED symptomatology or BD when asked to answer questions targeting body image and eating behavior. Possibly, these men were induced with negative emotions by the questions and thus decided to prematurely terminate the survey. In further studies, sample characteristics of prematurely terminating participants should be provided in detail. Also, a personal contact to the experimenter, e.g. via a diagnostic interview, may be not only useful to assess mental health of the participants but would also positively influence compliance.

To conclude, our model confirmed the role of TSF as a potential mediator in the relationship between $\mathrm{BD}$ and $\mathrm{DECB}$, but did not identify emotion dysregulation as a corresponding mediator. Overall, the model explained $43 \%$ of the variance in DECB of our non-clinical community sample. Even though the present findings have to be reassessed in larger, more representative samples and based on a longitudinal design, in the present sample of non-clinical young male participants TSF seems to represent a cognitive vulnerability that needs to be taken into account when describing underlying mechanisms associated with $\mathrm{BD}$ and disordered eating behavior. It is assumed that the 
susceptibility to TSF impacts self-related information processing and has emotional and behavioral consequences. While it has already been experimentally shown that in women food items (Coelho et al., 2008) and thin ideals (Wyssen et al., 2016) may provoke thought fusion linked to the experience of fear of weight gain, which leads to a vicious circle of negative affect and dysfunctional weight and shape regulation behavior, it has yet to be investigated, whether similar processes are present in men. Research is further needed to examine whether there are gender-specific triggers induce such cognitive processes and behavioral reactions in men (e.g., specific food items or muscularity ideals). 


\section{Acknowledgements}

Author JB was financially supported by the DocMobility grant of the Swiss National Science Foundation (Project Nr.: P1FRP_15562).

We thank lan Law for proof reading the article and Arabela Djurdjevic for her assistance with the preparation of the manuscript.

\section{Conflict of interest}

None. 


\section{References}

Allen, K.L., Byrne, S.M., Oddy, W.H., Crosby, R.D., 2013. DSM-IV-TR and DSM-5 eating disorders in adolescents: prevalence, stability, and psychosocial correlates in a population-based sample of male and female adolescents. J Abnorm Psychol 122 (3), 720-732.

Barlett, C.P., Vowels, C.L., Saucier, D.A., 2008. Meta-analyses of the effects of media images on men's body-image concerns. Journal of Social and Clinical Psychology 27 (3), 279-310.

Beck, A.T., Steer, R.A., Brown, G.K., 1996. Beck Depression Inventory-Second Edition manual. The Psychological Corporation, San Antonio, TX.

Blashill, A.J., 2014. A Dual Pathway Model of Steroid Use among Adolescent Boys: Results from a Nationally Representative Sample. Psychology of Men \& Masculinity 15 (2), 229-233.

Brower, K.J., Blow, F.C., Hill, E.M., 1994. Risk-Factors for Anabolic-Androgenic Steroid Use in Men. Journal of Psychiatric Research 28 (4), 369-380.

Cafri, G., Thompson, J.K., Ricciardelli, L., McCabe, M., Smolak, L., Yesalis, C., 2005. Pursuit of the muscular ideal: Physical and psychological consequences and putative risk factors. Clinical Psychology Review 25 (2), 215-239.

Coelho, J.S., Baeyens, C., Purdon, C., Shafran, R., Roulin, J.L., Bouvard, M., 2013. Assessment of thought-shape fusion: Initial validation of a short version of the trait thought-shape fusion scale. International Journal of Eating Disorders 46 (1), 77-85.

Coelho, J.S., Carter, J.C., McFarlane, T., Polivy, J., 2008. "Just looking at food makes me gain weight": experimental induction of thought-shape fusion in eating-disordered and non-eatingdisordered women. Behav Res Ther 46 (2), 219-228.

Coelho, J.S., Roefs, A., Jansen, A., 2010. The role of food-cue exposure and negative affect in the experience of thought-shape fusion. Journal of Behavior Therapy and Experimental Psychiatry 41 (4), 409-417.

Cooper, Z., Fairburn, C.G., 2011. The Evolution of "Enhanced" Cognitive Behavior Therapy for Eating Disorders: Learning From Treatment Nonresponse. Cogn Behav Pract 18 (3), 394-402.

Culpert, K.M., Racine, S.E., Klump, K.L., 2015. Research Review: What we have learned about the causes of eating disorders - a synthesis of sociocultural, psychological, and biological research. Journal of Child Psychology and Psychiatry 56 (11), 1141-1164.

Dakanalis, A., Clerici, M., Caslini, M., Gaudio, S., Serino, S., Riva, G., Carra, G., 2016. Predictors of initiation and persistence of recurrent binge eating and inappropriate weight compensatory behaviors in college men. International Journal of Eating Disorders.

Dakanalis, A., Favagrossa, L., Clerici, M., Prunas, A., Colmegna, F., Zanetti, M.A., Riva, G., 2015a. Body Dissatisfaction and Eating Disorder Symptomatology: A Latent Structural Equation Modeling Analysis of Moderating Variables in 18-to-28-Year-Old Males. Journal of Psychology 149 (1-2), 85-112.

Dakanalis, A., Timko, A., Madeddu, F., Volpato, C., Clerici, M., Riva, G., Zanetti, A.M., 2015b. Are the Male Body Dissatisfaction and Drive for Muscularity Scales reliable and valid instruments? J Health Psychol 20 (1), 48-59.

Dakanalis, A., Timko, C.A., Clerici, M., Zanetti, M.A., Riva, G., 2014a. Comprehensive examination of the trans-diagnostic cognitive behavioral model of eating disorders in males. Eating behaviors 15 (1), 63-67.

Dakanalis, A., Timko, C.A., Favagrossa, L., Riva, G., Zanetti, M.A., Clerici, M., 2014b. Why do only a minority of men report severe levels of eating disorder symptomatology, when so many report substantial body dissatisfaction? Examination of exacerbating factors. Eat Disord 22 (4), 292-305.

Dakanalis, A., Zanetti, A.M., Riva, G., Colmegna, F., Volpato, C., Madeddu, F., Clerici, M., 2015c. Male body dissatisfaction and eating disorder symptomatology: moderating variables among men. J Health Psychol 20 (1), 80-90. 
Di Pietro, M., Silveira, D.X., 2009. Internal validity, dimensionality and performance of the Body Shape Questionnaire in a group of Brazilian college students. Rev Bras Psiquiatr 31 (1), 21-24.

Dienes, Z., 2011. Bayesian Versus Orthodox Statistics: Which Side Are You On? Perspectives on Psychological Science 6 (3), 274-290.

Dubois, J.S., Altieri, T., Schembri, A., 2013. Thinking yourself fat: The perceived relationship between thoughts and body shape. Clinical Psychologist.

Ehring, T., Fischer, S., Schnulle, J., Bosterling, A., Tuschen-Caffier, B., 2008. Characteristics of emotion regulation in recovered depressed versus never depressed individuals. Personality and Individual Differences 44 (7), 1574-1584.

Evans, C., Dolan, B., 1993. Body Shape Questionnaire - Derivation of Shortened Alternate Forms. International Journal of Eating Disorders 13 (3), 315-321.

Fairburn, C.G., Beglin, S.J., 1994. Assessment of Eating Disorders - Interview or Self-Report Questionnaire. International Journal of Eating Disorders 16 (4), 363-370.

Fairburn, C.G., Cooper, Z., Shafran, R., 2003. Cognitive behaviour therapy for eating disorders: a "transdiagnostic" theory and treatment. Behav Res Ther 41 (5), 509-528.

Fiske, L., Fallon, E.A., Blissmer, B., Redding, C.A., 2014. Prevalence of body dissatisfaction among United States adults: Review and recommendations for future research. Eating behaviors 15 (3), 357-365.

Gotlib, I.H., 1984. Depression and general psychopathology in university students. J Abnorm Psychol 93 (1), 19-30.

Gratz, K.L., Roemer, L., 2004. Multidimensional assessment of emotion regulation and dysregulation: Development, factor structure, and initial validation of the difficulties in emotion regulation scale. Journal of Psychopathology and Behavioral Assessment 26 (1), 41-54.

Hautzinger, M., Keller, F., Kuehner, C., 2009. Beck Depressions-Inventar Revision (BDI-II). Manual. Frankfurt am Main: Pearson Assessment \& Information.

Hayes, A.F., 2012. PROCESS: A versatile computational tool for observed variable mediation, moderation, and conditional process modeling.

Hayes, S.C., Strosahl, K.D., Wilson, K.G., 1999. Acceptance and Commitment Therapy: An experiential approach to behavior change. . Guilford Press, New York.

Heatherton, T.F., Baumeister, R.F., 1991. Binge eating as escape from self-awareness. Psychol Bull $110(1), 86$.

Hilbert, A., Tuschen-Caffier, B., 2006. Eating Disorder Examination: Deutschsprachige Übersetzung. Verlag für Psychotherapie, Münster: .

Hilbert, A., Tuschen-Caffier, B., Karwautz, A., Niederhofer, H., Munsch, S., 2007. Eating disorder examination-questionnaire: Psychometric properties of the German version. Diagnostica 53 (3), 144-154.

Hudson, J.I., Hiripi, E., Pope, H.G., Kessler, R.C., 2007. The prevalence and correlates of eating disorders in the national comorbidity survey replication. Biological Psychiatry 61 (3), 348-358.

Jankowski, G.S., Diedrichs, P.C., Halliwell, E., 2014. Can appearance conversations explain differences between gay and heterosexual men's body dissatisfaction? Psychology of Men \& Masculinity $15(1), 68$.

Kühner, C., Bürger, C., Keller, F., Hautzinger, M., 2007. Reliabilität und validität des revidierten beckdepressionsinventars (BDI-II). Der Nervenarzt 78 (6), 651-656.

Lavender, J.M., Anderson, D.A., 2010. Contribution of Emotion Regulation Difficulties to Disordered Eating and Body Dissatisfaction in College Men. International Journal of Eating Disorders 43 (4), 352-357.

Lavender, J.M., De Young, K.P., Anderson, D.A., 2010. Eating Disorder Examination Questionnaire (EDE-Q): norms for undergraduate men. Eating behaviors 11 (2), 119-121.

McCreary, D.R., Sasse, D.K., Saucier, D.M., Dorsch, K.D., 2004. Measuring the Drive for Muscularity: Factorial Validity of the Drive for Muscularity Scale in Men and Women. Psychology of Men \& Masculinity 5 (1), 49. 
Merwin, R.M., Timko, C.A., Moskovich, A.A., Ingle, K.K., Bulik, C.M., Zucker, N.L., 2011. Psychological inflexibility and symptom expression in anorexia nervosa. Eat Disord 19 (1), 62-82.

Mitchison, D., Mond, J.M., 2015. Epidemiology of eating disorders, eating disordered behaviour, and body image disturbance in males: A narrative review. Journal of Eating Disorders 3, 20.

Mond, J.M., Hay, P.J., Rodgers, B., Owen, C., Beumont, R.J.V., 2004. Validity of the Eating Disorder Examination Questionnaire (EDE-Q) in screening for eating disorders in community samples. Behav Res Ther 42 (5), 551-567.

Naumann, E., Tuschen-Caffier, B., Voderholzer, U., Caffier, D., Svaldi, J., 2015. Rumination but not distraction increases eating-related symptoms in anorexia and bulimia nervosa. J Abnorm Psychol 124 (2), 412-420.

Neumark-Sztainer, D., Paxton, S.J., Hannan, P.J., Haines, J., Story, M., 2006. Does body satisfaction matter? Five-year longitudinal associations between body satisfaction and health behaviors in adolescent females and males. Journal of Adolescent Health 39 (2), 244-251.

Nolen-Hoeksema, S., 2012. Emotion Regulation and Psychopathology: The Role of Gender. Annual Review of Clinical Psychology 8, 161-187.

Pook, M., Tuschen-Caffier, B., Brahler, E., 2008. Evaluation and comparison of different versions of the Body Shape Questionnaire. Psychiatry Res 158 (1), 67-73.

Pook, M., Tuschen-Caffier, B., Stich, N., 2002. Evaluation of the 'Fragebogen zum Figurbewusstsein' (FFB, German version of the Body Shape Questionnaire). Verhaltenstherapie 12 (2), 116-124.

Preti, A., de Girolamo, G., Vilagut, G., Alonso, J., de Graaf, R., Bruffaerts, R., Demyttenaere, K., PintoMeza, A., Haro, J.M., Morosini, P., Investigators, E.-W., 2009. The epidemiology of eating disorders in six European countries: Results of the ESEMeD-WMH project. Journal of Psychiatric Research 43 (14), 1125-1132.

Probst, M., Pieters, G., Vanderlinden, J., 2009. Body experience assessment in non-clinical male and female subjects. Eating and Weight Disorders 14 (1), e16-21.

Rachman, S., Shafran, R., 1999. Cognitive distort ions: Thought-action fusion. Clinical Psychology \& Psychotherapy 6 (2), 80-85.

Radomsky, A.S., de Silva, P., Todd, G., Treasure, J., Murphy, T., 2002. Thought-shape fusion in anorexia nervosa: an experimental investigation. Behav Res Ther 40 (10), 1169-1177.

Raevuori, A., Keski-Rahkonen, A., Hoek, H.W., 2014. A review of eating disorders in males. Curr Opin Psychiatry 27 (6), 426-430.

Ricciardelli, L.A., McCabe, M.P., 2003. Sociocultural and individual influences on muscle gain and weight loss strategies among adolescent boys and girls. Psychology in the Schools 40 (2), 209224.

Rosen, J.C., Jones, A., Ramirez, E., Waxman, S., 1996. Body Shape Questionnaire: studies of validity and reliability. International Journal of Eating Disorders 20 (3), 315-319.

Rosseel, Y., 2012. lavaan: An R package for structural equation modeling. Journal of Statistical Software 48 (2), 1-36.

Shafran, R., Robinson, P., 2004. Thought-shape fusion in eating disorders. British Journal of Clinical Psychology 43, 399-408.

Shafran, R., Teachman, B.A., Kerry, S., Rachman, S., 1999. A cognitive distortion associated with eating disorders: Thought-shape fusion. British Journal of Clinical Psychology 38, 167-179.

Shafran, R., Thordarson, D.S., Rachman, S., 1996. Thought-action fusion in obsessive compulsive disorder. Journal of Anxiety Disorders 10 (5), 379-391.

Stice, E., 2001. A prospective test of the dual-pathway model of bulimic pathology: mediating effects of dieting and negative affect. J Abnorm Psychol 110 (1), 124-135.

Stice, E., 2002. Risk and maintenance factors for eating pathology: a meta-analytic review. Psychol Bull 128 (5), 825-848.

Stice, E., Marti, C.N., Durant, S., 2011. Risk factors for onset of eating disorders: evidence of multiple risk pathways from an 8-year prospective study. Behav Res Ther 49 (10), 622-627. 
Stice, E., Shaw, H.E., 2002. Role of body dissatisfaction in the onset and maintenance of eating pathology - A synthesis of research findings. Journal of Psychosomatic Research 53 (5), 985993.

Svaldi, J., Griepenstroh, J., Tuschen-Caffier, B., Ehring, T., 2012. Emotion regulation deficits in eating disorders: a marker of eating pathology or general psychopathology? Psychiatry Res 197 (12), 103-111.

Thompson, J.K., Cafri, G., 2007. The Muscular Ideal: Psychological, Social and Medical Perspectives. American Psychological Association., Washington, DC.

Tylka, T.L., 2011. Refinement of the tripartite influence model for men: Dual body image pathways to body change behaviors. Body Image 8 (3), 199-207.

Tylka, T.L., Bergeron, D., Schwartz, J.P., 2005. Development and psychometric evaluation of the Male Body Attitudes Scale (MBAS). Body Image 2 (2), 161-175.

Woodside, D.B., Garfinkel, P.E., Lin, E., Goering, P., Kaplan, A.S., Goldbloom, D.S., Kennedy, S.H., 2001. Comparisons of men with full or partial eating disorders, men without eating disorders, and women with eating disorders in the community. American Journal of Psychiatry 158 (4), 570-574.

Wyssen, A., Coelho, J.S., Wilhelm, P., Zimmermann, G., Munsch, S., 2016. Thought-shape fusion in young healthy females appears after vivid imagination of thin ideals. Journal of Behavior Therapy and Experimental Psychiatry 52, 75-82. 


\section{Tables and figures}

FIGURE 1: THE MEDIATION MODEL OF DISTURBED EATING IN MEN.

Note. Standardized regression coefficients. Direct effect of body dissatisfaction (BD) to disturbed eating and compensatory behavior (DECB) $($ Beta $=0.43, S E=0.09, z=4.67, p<0.001)$. Global indirect effect: BD - (Thought-Shape Fusion \& Emotion dysregulation) - DECB (Beta = 0.56, SE =0.08, z= 7.09, $p<0.001)$. The covariance between emotion dysregulation and thought-shape fusion is 0.10 (SE = $0.06, z=1.77, p=0.08) .{ }^{* * *}=p<0.001$.

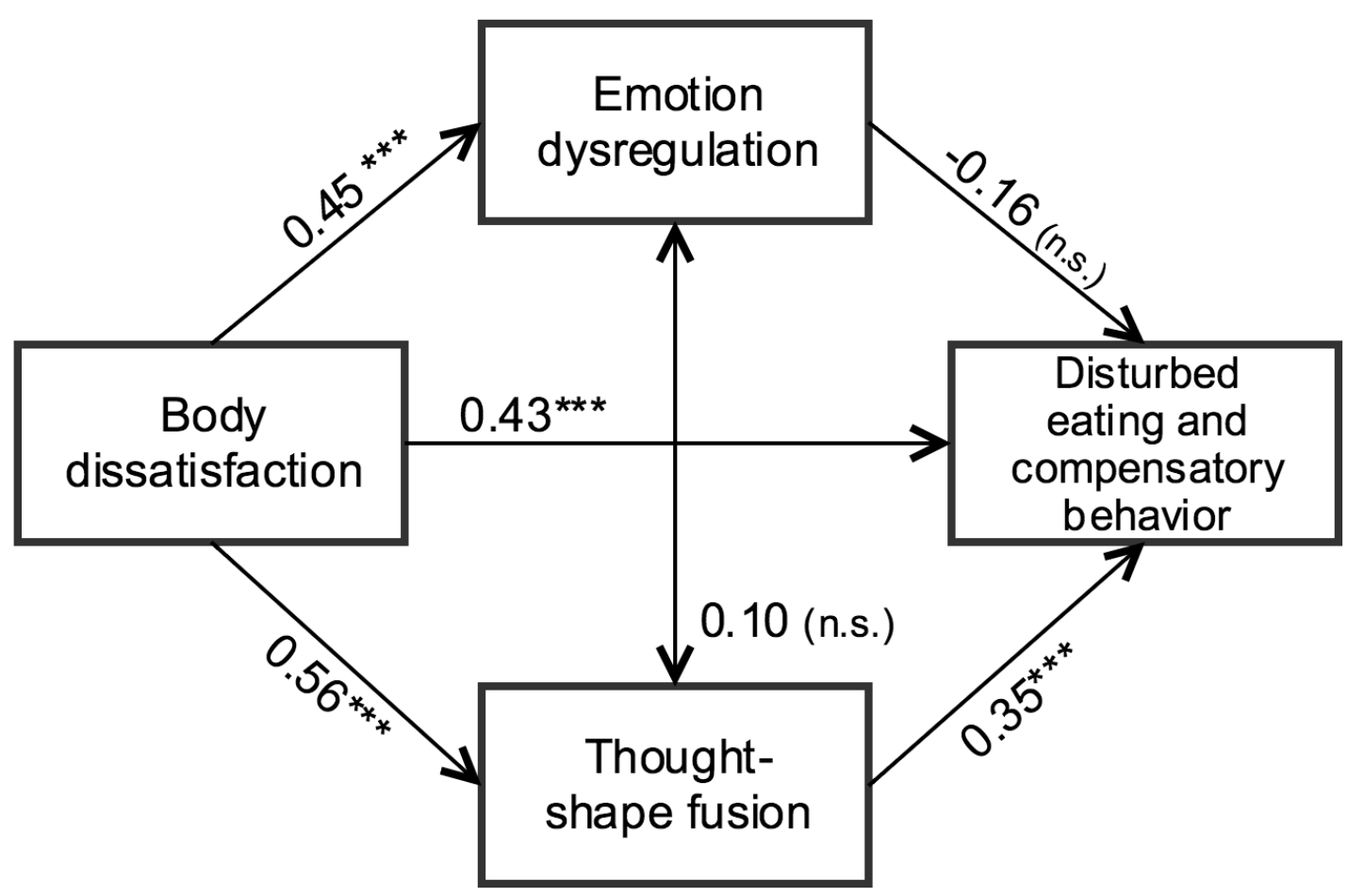


TABLE 1. DESCRIPTIVE STATISTICS. Range, means, standard deviations, and cut-off values for all variables involved in the study.

\begin{tabular}{llllc}
\hline Variable & Range & Mean & SD & Cut-off \\
\hline Age & $18-37$ & 23.75 & 3.17 & - \\
BMI & $18.22-41.58$ & 23.27 & 3.05 & $18.5<x>25^{1}$ \\
BDI-II & $0-38$ & 5.98 & 6.74 & $>13^{2}$ \\
EDE-Q global & $0-3.91$ & 0.53 & 0.63 & $>2.09^{3}$ \\
EDE-Q DECB & $0-4.22$ & 0.35 & 0.61 & - \\
BSQ short & $8-39$ & 12.85 & 6.02 & $>18.00^{4}$ \\
DERS global & $37-141$ & 78.50 & 20.43 & $>99.45^{5}$ \\
TSF & $0-28$ & 2.99 & 5.49 & - \\
\hline
\end{tabular}

Note: $\mathrm{SD}=$ standard deviation; 1) BMI = Body Mass Index; Normal weight according to the World Health Organization (WHO); 2) BDI-II = Beck Depression Inventory; Values from 0-13 refer to none or minimal, 14-19 to mild, 20-28 to moderate, and 29 or above to severe depressive symptoms (Hautzinger et al., 2009); 3) EDE-Q = Eating Disorder Examination Questionnaire; Mean plus one standard deviation according to (Lavender et al., 2010); 4) BSQ = Body Shape Questionnaire; cut-off according to (Evans and Dolan, 1993); 5) DERS = Difficulties in Emotion Regulation Scale; Mean plus one standard deviation according to (Gratz and Roemer, 2004); TSF = Thought-Shape Fusion Scale. 
TABLE 2. RESULTS OF THE PATH ANALYSIS testing the coefficients of the proposed Model of Disturbed Eating in Men ( $\mathrm{N}=123)$. Note: standardized coefficients, bootstrapped by $\mathrm{N}=5000$.

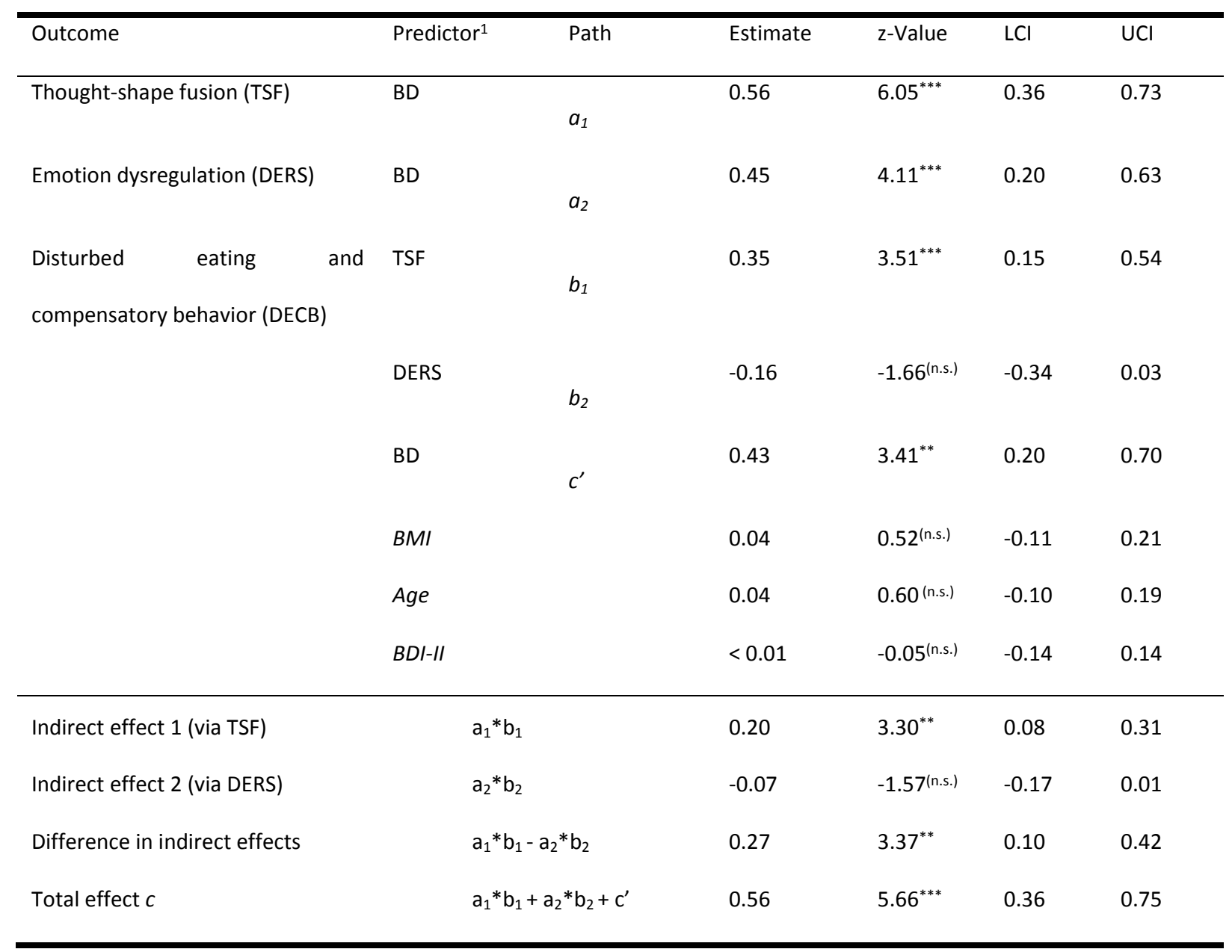

${ }^{1}$ Terms written in italics denote covariates.

${ }^{* * *}=p<0.001 ; * *=p<0.01 ;$ n.s. $=$ non-significant; $\mathrm{DECB}=$ Disturbed Eating and Compensatory Behavior; $\mathrm{BD}=\mathrm{Body}$ Dissatisfaction; TSF = Thought-Shape Fusion; BDI = Beck Depression Inventory; DERS = Difficulties in Emotion Regulation Scale; $\mathrm{LCl} / \mathrm{UCl}=$ lower/upper confidence interval. 